Original Research Article

\title{
Structural sensitivity and resilience in a predator-prey model with density-dependent mortality
}

\author{
C. Aldebert *, D. Nerini, M. Gauduchon, J.C. Poggiale \\ Mediterranean Institute of Oceanography, Aix-Marseille University, Toulon University, CNRS/INSU, IRD, MIO, UM 110, 13288 Marseille, Cedex 09, France
}

\section{A R T I C L E I N F O}

\section{Article history:}

Received 8 July 2015

Received in revised form 18 May 2016

Accepted 25 May 2016

Available online 4 June 2016

\section{Keywords:}

Structural sensitivity

Functional response

Bifurcations

Generalized modelling

Multiple stable states

Hysteresis

\begin{abstract}
A B S T R A C T
Numerous formulations with the same mathematical properties can be relevant to model a biological process. Different formulations can predict different model dynamics like equilibrium vs. oscillations even if they are quantitatively close (structural sensitivity). The question we address in this paper is: does the choice of a formulation affect predictions on the number of stable states? We focus on a predator-prey model with predator competition that exhibits multiple stable states. A bifurcation analysis is realized with respect to prey carrying capacity and species body mass ratio within range of values found in food web models. Bifurcation diagrams built for two type-II functional responses are different in two ways. First, the kind of stable state (equilibrium vs. oscillations) is different for 26.0$49.4 \%$ of the parameter values, depending on the parameter space investigated. Using generalized modelling, we highlight the role of functional response slope in this difference. Secondly, the number of stable states is higher with Ivlev's functional response for 0.1-14.3\% of the parameter values. These two changes interact to create different model predictions if a parameter value or a state variable is altered. In these two examples of disturbance, Holling's disc equation predicts a higher system resilience. Indeed, Ivlev's functional response predicts that disturbance may trap the system into an alternative stable state that can be escaped from only by a larger alteration (hysteresis phenomena). Two questions arise from this work: (i) how much complex ecological models can be affected by this sensitivity to model formulation? and (ii) how to deal with these uncertainties in model predictions?
\end{abstract}

(c) 2016 Elsevier B.V. All rights reserved.

\section{Introduction}

The choice of a model formulation in biology is often associated to uncertainties. Uncertainties arise from intrinsic data variability and simplified assumptions chosen to represent complex processes. Numerous mathematical formulations of a process are relevant in the sense that: (i) they fit empirical data, (ii) their properties and assumptions are consistent with the knowledge of the studied system (Mullin et al., 1975; Cordoleani et al., 2011). Even if these functions are quantitatively close, they can predict very different model dynamics (Myerscough et al., 1996; Wood and Thomas, 1999; Gross et al., 2004; Fussmann and Blasius, 2005; Poggiale et al., 2010; Adamson and Morozov, 2012, 2014). This change in model dynamics can be both quantitative and qualitative, a phenomenon coined "structural sensitivity" (Cordoleani et al., 2011).

\footnotetext{
* Corresponding author.

E-mail addresses: clement.aldebert@univ-amu.fr (C. Aldebert), david.nerini@univ-amu.fr (D. Nerini), mathias.gauduchon@univ-amu.fr (M. Gauduchon), jean-christophe.poggiale@univ-amu.fr (J.C. Poggiale).
}

Structural sensitivity has been mainly explored in models of predator-prey interactions. Predation emerges from the interplay between physiological, individual and collective processes. Depending on which processes are considered, predation can be modelled using numerous functional responses (amount of prey eaten per predator and per time unit, see Jeschke et al., 2002; Gentleman et al., 2003, for a review). Functional responses are classified by their main mathematical properties that define different types, such as Holling-types (1959a) or with vs. without predator interference (Beddington, 1975; DeAngelis et al., 1975). Two functions of different type create different dynamics (Cantrell and Cosner, 2001; Oaten and Murdoch, 1975; Scheffer and de Boer, 1995). But different dynamics are also generated by functions that belong to the same type. A model is thus structurally sensitive to the functional response formulation. For example, different type-II functional responses predict either a stable equilibrium or oscillations in predator-prey and food chain models. These models are also more sensitive to functional response formulation than to parameter values (Myerscough et al., 1996; Gross et al., 2004; Fussmann and Blasius, 2005; Cordoleani et al., 2011; Adamson and Morozov, 2012, 2014). 
To overcome both parameter and structural sensitivity, Gross and Feudel (2006) proposed a method called generalized modelling. The local stability of positive equilibria is studied in a class of models without specifying their exact formulation and parameter values (see Yeakel et al., 2011, for a review in ecology). New parameters are defined to describe system dynamics near an equilibrium. As a drawback, this method is local and cannot explore global situations as a whole, like multiple stable states.

Multiple stable states can be important to investigate how a system behaves when facing some disturbances. Thus, the study of multiple stable states is of growing interest in ecology (Beisner et al., 2003; Knowlton, 2004; Scheffer et al., 2009, 2012). Despite this interest, studies on structural sensitivity focused on qualitative change (equilibrium vs. limit cycle) of a single stable state (except a short note in Fussmann and Blasius, 2005). The number of stable states can be modified by a quantitatively small change in model formulation in theory (as discussed by Adamson and Morozov (2014)), but such possibility has not been investigated so far. However, multiple stable states can coexist in predator-prey models like Bazykin's model (Bazykin et al., 1985, in Metzler and Wischniewsky, 1985; Kuznetsov, 2004).

Bazykin's model is equivalent to Rosenzweig and MacArthur's model (1963) with density-dependent mortality for the predator. The predator has no density-dependent mortality in previous studies on structural sensitivity and generalized predator-prey models (Kuehn and Gross, 2011; Yeakel et al., 2011). However, density-dependent mortality represents the effects of diseases and/ or competition and can be relevant for a wide range of predator species (Loreau, 2010). Furthermore, a density-dependent mortality is often used for the top-most predator in applied ecological models as a closure term to implicitly represent higher trophic levels (Fulton et al., 2003a,b). Predator competition modelled with quadratic mortality implicitly involves other limiting resource than the prey. In case of predator interference, the functional response may be predator-dependent (Ivlev, 1955; Beddington, 1975; DeAngelis et al., 1975; Arditi and Ginzburg, 1989; DeAngelis, 2013). However, different predator-dependent functional responses exist and structural sensitivity can also be studied in models based on this type of functions.

The question we want to address in this paper is: what is the impact of structural sensitivity on the number of stable states? We focus on Bazykin's model which can exhibit multiple stable states. This predator-prey model can be a building block of some food web models (Aldebert et al., submitted for publication; Plitzko et al., 2012, and references therein) and its study may help to understand those more complex models. The next section presents Bazykin's model and the functional response formulations that we test. Then a bifurcation analysis is conducted for two functional response formulations. In the fourth section, we derive a generalized predator-prey model in order to identify stabilizing factors independently of a specific formulation. This provides an additional understanding of the local stability of equilibria found in the previous section. Finally, results are discussed using examples where system resilience predicted by the model is tested using different functional response formulations.

\section{Predator-prey model}

We modelled predator-prey dynamics with Bazykin's model. We wrote the model in a form that can easily be extended to more complex food webs. Population dynamics are modelled using the following differential system:

$$
\left\{\begin{array}{l}
\frac{d B_{\text {prey }}}{d t}=\left[\lambda q^{\phi}-\alpha_{\text {prey }}-\omega \beta_{\text {prey }} B_{\text {prey }}\right] B_{\text {prey }}-G^{\phi}\left(B_{\text {prey }}\right) B_{\text {pred }} \\
\frac{d B_{\text {pred }}}{d t}=\left[\lambda G^{\phi}\left(B_{\text {prey }}\right)-\alpha_{\text {pred }}-\beta_{\text {pred }} B_{\text {pred }}\right] B_{\text {pred }},
\end{array}\right.
$$

where $B_{\text {prey }}$ and $B_{\text {pred }}$ are the respective biomass of unstructured prey and predator populations. In model (1), the prey grows using an implicit constant resource with a rate $q^{\phi}$. The predator feeds on the prey with a functional response $G^{\phi}\left(B_{\text {prey }}\right)$. We assume that both populations have the same conversion efficiency $\lambda$. Each population has intrinsic losses due to (i) linear mortality with a mortality rate $\alpha_{\text {prey }}$ (resp. $\alpha_{\text {pred }}$ ) and (ii) competition with a per-capita density-dependent mortality rate $\beta_{\text {prey }}$ (resp. $\beta_{\text {pred }}$ ). Prey competition is proportional to an environmental parameter $\omega$, so prey carrying capacity is proportional to $1 / \omega$. Predation is modelled using a type-II functional response $G^{\phi}$ which does not depend on predator biomass and fulfills the following properties:

$$
\begin{gathered}
G^{\phi} \in \mathcal{C}^{2}, \quad G^{\phi}(0)=0, \quad G^{\phi}\left(B_{\text {prey }}\right) \geq 0, \quad G^{\phi^{\prime}}\left(B_{\text {prey }}\right)>0, \\
G^{\phi^{\prime \prime}}\left(B_{\text {prey }}\right)<0, \quad \lim _{B_{\text {prey }} \rightarrow+\infty} G^{\phi}\left(B_{\text {prey }}\right)<+\infty,
\end{gathered}
$$

where $\mathcal{C}^{2}$ is the class of twice continuously differentiable functions. Other properties means that $G^{\phi}$ is null in absence of prey, increases with prey biomass, is concave and saturates at high prey biomass.

As examples of functions with properties (2), we consider Holling's disc equation (1959b, 1965) $G^{H}$ and Ivlev's functional response (1955) $G^{I}$ (3) (Fig. 1):

$$
\begin{aligned}
G^{H}\left(B_{\text {prey }}\right) & =\frac{a_{\text {pred }}^{H} B_{\text {prey }}}{1+h_{\text {pred }}^{H} a_{\text {pred }}^{H} B_{\text {prey }}}, \\
G^{I}\left(B_{\text {prey }}\right) & =\frac{1}{h_{\text {pred }}^{I}}\left(1-\exp \left(-h_{\text {pred }}^{I} a_{\text {pred }}^{I} B_{\text {prey }}\right)\right) .
\end{aligned}
$$

For the first formulation, parameters $a_{\text {pred }}^{H}$ and $h_{\text {pred }}^{H}$ are respectively the attack rate and the handling time of the predator. For the second formulation, parameter $1 / h_{\text {pred }}^{I}$ is the maximal consumption rate and $a_{\text {pred }}^{I} h_{\text {pred }}^{I}$ is the satiation coefficient of the predator. Parameters are defined in order to have a consistent mathematical meaning across formulations (4):

$G^{\phi^{\prime}}(0)=a_{\text {pred }}^{\phi}, \quad \lim _{B_{\text {prey }} \rightarrow+\infty} G^{\phi}\left(B_{\text {prey }}\right)=\frac{1}{h_{\text {pred }}^{\phi}}$.

Thus, $a_{\text {pred }}^{\phi}$ gives the slope of the functional response at the origin, and $1 / h_{\text {pred }}^{\phi}$ gives the asymptotic value of the functional response when it saturates at high prey biomass.

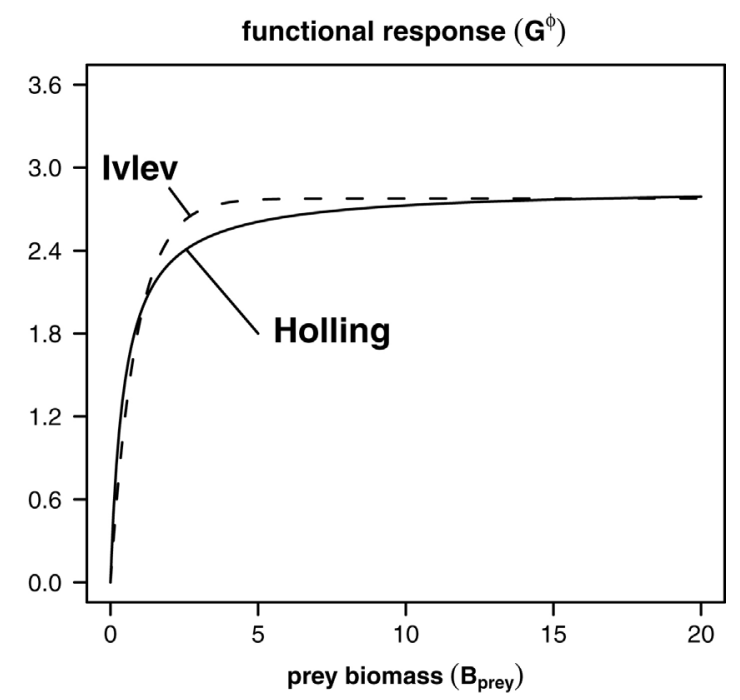

Fig. 1. Functional responses used in the model: Holling's disc equation (solid) and best fitted Ivlev's functional response (dashed). The former is used as "data" to parameterize the latter (Section 1 in supplementary material). Parameter values are given in Table 1 . For the sake of visibility, only a part of the fitting range ([0, $\left.\left.B_{\text {res }}\right]=[0,500]\right)$ is shown. 
Resource consumption $q^{\phi}$ (5) has the same equation as the functional response $G^{\phi}$ with a constant pool of resource $B_{\text {res }}$ :

$q^{H}=\frac{a_{\text {prey }}^{H} B_{\text {res }}}{1+h_{\text {prey }}^{H} a_{\text {prey }}^{H} B_{\text {res }}}, \quad q^{I}=\frac{1}{h_{\text {prey }}^{I}}\left(1-\exp \left(-h_{\text {prey }}^{I} a_{\text {prey }}^{I} B_{\text {res }}\right)\right)$,

so $q^{\phi}$ is a constant. Organism's metabolic rates are strongly influence by body mass. We assume that some parameter values scale allometrically with species body mass $M_{\text {prey }}$ and $M_{\text {pred }}$ (Brown et al., 2004; Kooijman, 2010):

$$
\begin{array}{cl}
a_{i}^{\phi}=a^{\phi} M_{i}^{-0.25}, & h_{i}^{\phi}=h^{\phi} M_{i}^{0.25}, \quad \alpha_{i}=\alpha M_{i}^{-0.25}, \\
\beta_{i}=\beta M_{i}^{-0.25} & \text { with } i=\text { prey,pred. }
\end{array}
$$

These relationships imply an allometric scaling of resource consumption $q^{\phi} \propto M_{\text {prey }}^{-0.25}$ and predation $G^{\phi} \propto M_{\text {pred }}^{-0.25}$. So we define $q^{\phi}:=q^{\phi} M_{\text {prey }}^{0.25}$ and $G^{\phi}:=G^{\phi} M_{\text {pred }}^{0.25}$, which do not depend on species body mass. Hence, model (1) can be written using body masses:

$$
\left\{\begin{array}{l}
\frac{d B_{\text {prey }}}{d t-}=\left[\lambda q^{\phi}-\alpha-\omega \beta B_{\text {prey }}\right] B_{\text {prey }}-G^{\phi}\left(B_{\text {prey }}\right) B_{\text {pred }}\left(M_{\text {pred }} / M_{\text {prey }}\right)^{-0.25} \\
\frac{d B_{\text {pred }}}{d t-}=\left[\lambda G^{\phi}\left(B_{\text {prey }}\right)-\alpha-\beta B_{\text {pred }}\right] B_{\text {pred }}\left(M_{\text {pred }} / M_{\text {prey }}\right)^{-0.25},
\end{array}\right.
$$

with a time re-scaling $t=t M_{\text {prey }}^{-0.25}$ in order to have a model that does not depend on both species body mass but on their ratio $M_{\text {pred }} / M_{\text {prey }}$. When Holling's disc equation is used, model (7) can be written in the form of Bazykin's model (Bazykin et al., 1985, in Metzler and Wischniewsky, 1985; Kuznetsov, 2004) after appropriate re-scaling and parameter changes.

Parameter values are listed in Table 1. To parameterize Ivlev's functional response, we consider Holling's disc equation as "data" and optimize Ivlev's parameters in order to minimize the weighted Euclidean distance between the two functional responses (see Section 1 in Supporting Online Material for details). This optimization is done to simulate a fit of both functional responses on empirical data (as done by Mullin et al., 1975; Cordoleani et al., 2011). To do this, the optimization step gives a better fit between functional responses (correlation coefficient $\rho=0.93$ ) than using the same $a^{\phi}$ and $h^{\phi}$ values for both formulations ( $\left.\rho=0.86\right)$, as done for example by Anderson et al. (2010).

\section{Bifurcation analysis for different functional responses}

Model (7) has two trivial equilibria (8):

$O=(0,0)$ and $E_{0}=\left(K:=\frac{\lambda q^{\phi}-\alpha}{\omega \beta}, 0\right)$, where $K$ is prey carrying capacity. Equilibrium $O$ always exists and is a saddle. Equilibrium $E_{0}$ exists if $\lambda q^{\phi}>\alpha$. It is a stable node if $\alpha>\lambda G^{\phi}(K)$ (Fig. 2, phase portrait 0 ) and a saddle otherwise (other phase portraits). The threshold $\alpha=\lambda G^{\phi}(K)$ corresponds to a transcritical bifurcation where a stable positive equilibrium appears when $1 / \omega$ is increasing. The twelve generic phase portraits found for this system are presented in Fig. 2. Briefly, the system exhibits up to three positive equilibria (phase portraits 3, 4 and 611) due to the density-dependent mortality of the predator, as well as two limit cycles (phase portraits 7 and 11). Multiple attractors co-exist in seven phase portraits (phase portraits 4-8, 10 and 11).

An analysis of model (1) with Holling's disc equation is presented in Kuznetsov (2004), based on a two-dimensional bifurcation diagram with respect to the saturation parameter of the functional response and the density-dependent mortality of the predator. Here we focus on two parameters that are expected to vary across ecosystems and species: (i) prey carrying capacity $(\propto 1 / \omega)$ and (ii) species body mass ratio $\left(M_{\text {pred }} / M_{\text {prey }}\right)$. In our analysis, body mass ratio ranges from prey that are $10^{4}$ times bigger than their predators (e.g. insects feeding on trees) to predators that are $10^{8}$ times bigger than their prey (e.g. whales feeding on krill). This range is likely to include most of known predator-prey interactions (Kooijman, 2010). For prey carrying capacity, $1 / \omega$ can be understood as a number of different trophic species eaten by the same predator species in a food web (Aldebert et al., submitted for publication). In our analysis, $1 / \omega$ ranges from 0 to 10 . This range includes almost all values observed in modelbased food webs that are consistent with empirical data (e.g. Williams and Martinez, 2000). The sensitivity of our results to parameter ranges will be discussed at the end of the section.

Two-dimensional bifurcation diagrams in the plane $(1 / \omega$, $\left.\log _{10}\left(M_{\text {pred }} / M_{\text {prey }}\right)\right)$ were obtained using the Matlab continuations toolboxes Matcont and CL_Matcont (Dhooge et al., 2006). Codimension-one (here curves) and codimension-two (here points) bifurcations were numerically computed using continuation methods (Kuznetsov, 2004). Homoclinic bifurcation curves were estimated by dichotomy for numerous parameter values. Fig. 3 presents the bifurcation diagrams obtained for both functional responses.

When Holling's disc equation is used (Fig. 3a), more than 99.999\% of the explored parameter space area generate phase portraits 1,2 or 3 . These portraits correspond respectively to a stable equilibrium, an unstable focus with a stable limit cycle and three equilibria (one unstable node or focus, a saddle, one stable node or focus). The area with three equilibria is delimited by the two branches of a saddle-node bifurcation (red curve). Transition between phase portraits 1 and 2 occurs through a Hopf bifurcation, which is either supercritical (dark blue curve) or subcritical (light blue curve). These two branches meet at a Bautin point on this

\begin{tabular}{|c|c|c|c|c|}
\hline Biological meaning & Parameter & Value & Source & Unit \\
\hline Mortality rate & $\alpha$ & 0.3 & (Heckmann et al., 2012) & time $^{-1}$ \\
\hline Per-capita competition rate & $\beta$ & 0.5 & (Heckmann et al., 2012) & biomass $^{-1}$ time $^{-1}$ \\
\hline Assimilation efficiency & $\lambda$ & 0.65 & (Heckmann et al., 2012) & - \\
\hline Resource biomass & $B_{\text {res }}$ & 500 & (Heckmann et al., 2012) & biomass \\
\hline \multicolumn{5}{|l|}{ Holling's disc equation: } \\
\hline Attack rate & $a^{H}$ & 6 & (Heckmann et al., 2012) & biomass $^{-1}$ time $^{-1}$ \\
\hline Handling time & $h^{H}$ & 0.35 & (Heckmann et al., 2012) & time \\
\hline \multicolumn{5}{|l|}{ Ivlev's functional response: } \\
\hline Maximal consumption rate & $1 / h^{I}$ & $1 / 0.36$ & see supplementary material & time $^{-1}$ \\
\hline Satiation coefficient & $a^{I} h^{I}$ & $3.17 \times 0.36$ & see supplementary material & biomass $^{-1}$ \\
\hline
\end{tabular}

Table 1

Parameter values used in the predator-prey model.

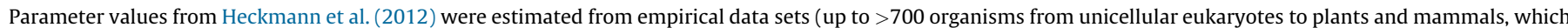

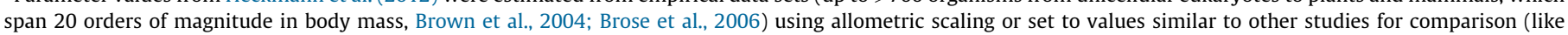
Kartascheff et al., 2009, 2010). 

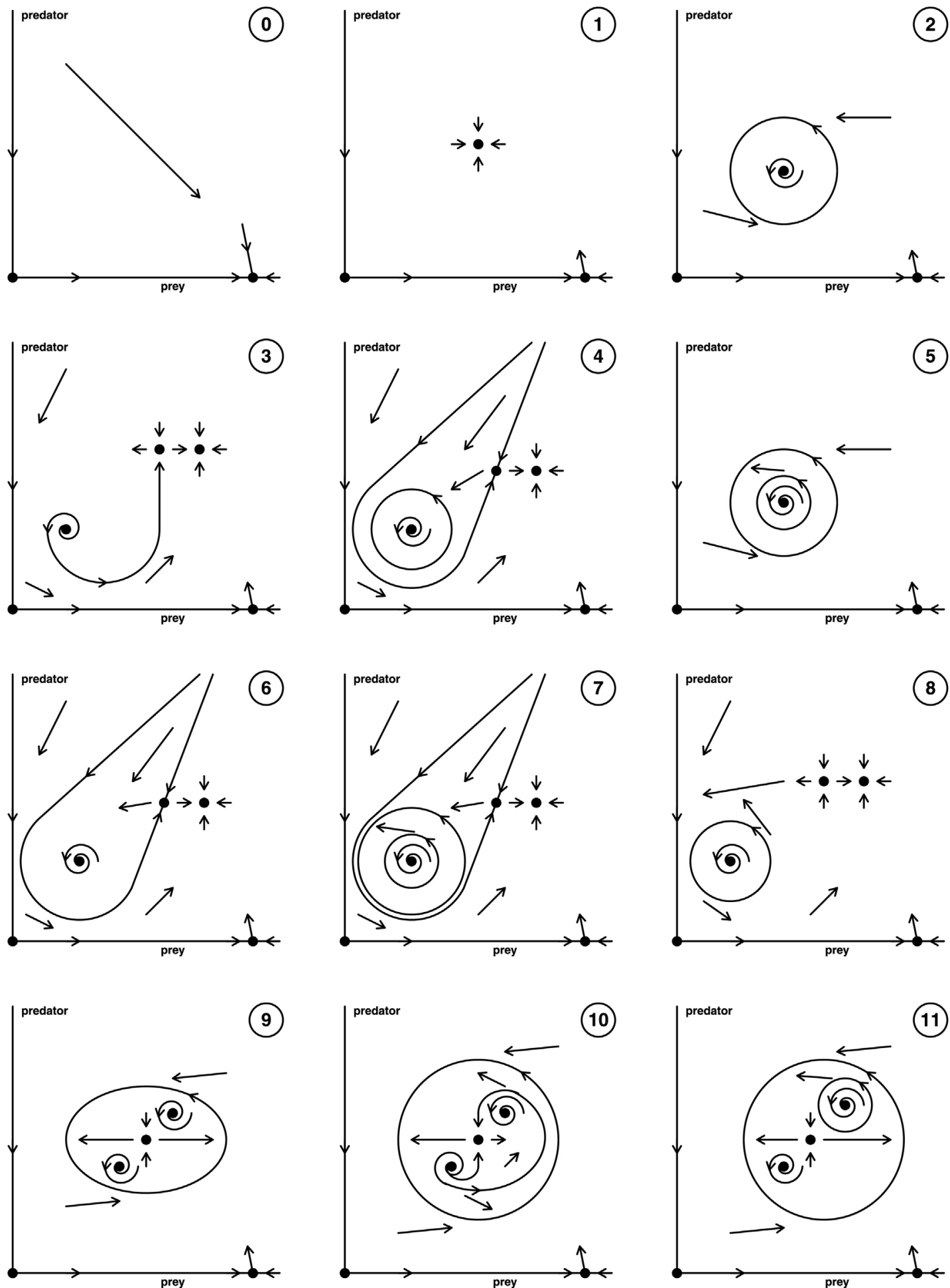

Fig. 2. Qualitative generic phase portraits of the predator-prey model. Transitions between the different situations corresponds to bifurcations drawn in Fig. 3.

curve. A limit point of cycles bifurcation curve (green curve) starts from this point and stays mostly close to the subcritical Hopf branch. When moving from phase portrait 1 to 2 through this branch and the limit point of cycles, the system exhibits phase portrait 5 in a tiny strip of the parameter space (stable focus and limit cycle separated by an unstable limit cycle). The two branches of the saddle-node bifurcation curves meet at a Cusp bifurcation point. The subcritical branch of the Hopf bifurcation curves meets the bottom-branch of the saddle-node curve at a Bogdanov-Takens point. Close to these points, there are three positive equilibria surrounded by a stable limit cycle (phase portraits 9-11). From phase portrait 2 to 3 , the limit cycle is destroyed through a homoclinic bifurcation (dashed black curve), which may be a homoclinic to saddle-node bifurcation in most of the bifurcation diagram (see Section 2 in supplementary material for a more detailed technical discussion).

If Ivlev's functional response is used (Fig. 3b), the BogdanovTakens point is now on the top-branch of the saddle-node 

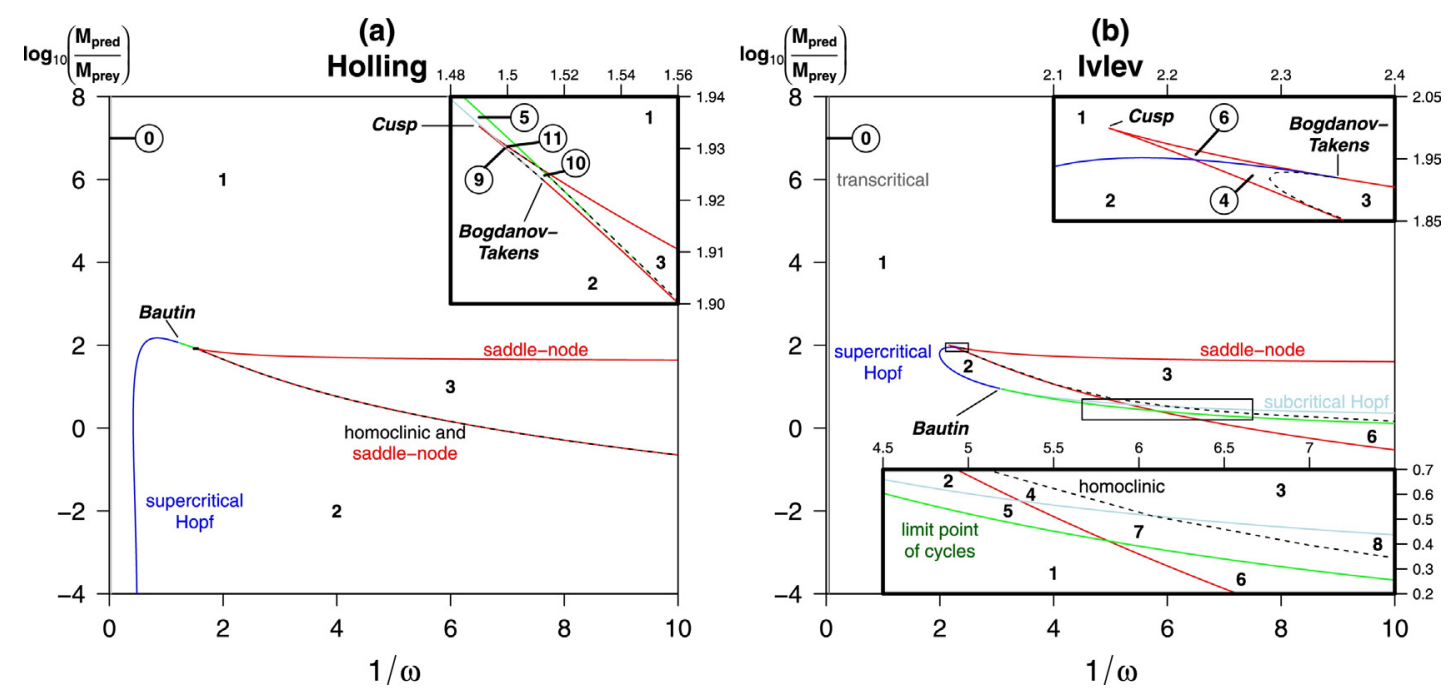

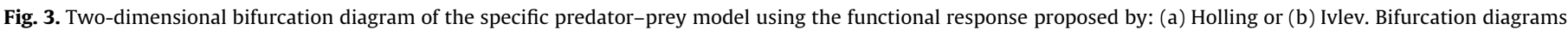

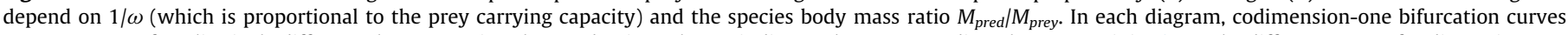

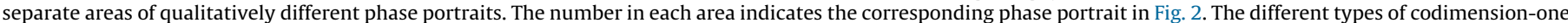

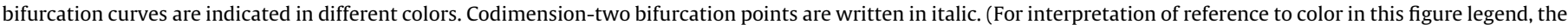
reader is referred to the web version of this article.)

bifurcation.The homoclinic curve starting from this point is close to the bottom branch of the saddle-node curve in most of the bifurcation diagram (see Section 2 in supplementary material). Starting from the Bogdanov-Takens point, the Hopf bifurcation is supercritical. In comparison with Holling's disc equation, the Hopf bifurcation curve wraps, which strongly decreases the part of the parameter space in which a stable limit cycle exists. This curve becomes a subcritical Hopf bifurcation after the Bautin point. A limit point of cycles curve starts from this Bautin point. As a consequence, various dynamics arise with Ivlev's functional response (see Fig. 3b, bottom zoom). For example, in phase portrait 7 (Fig. 2) the system has three different attractors: a stable focus, a stable limit cycle and a stable node. Their respective attraction basins are delimited by an unstable limit cycle and a saddle. Phase portraits 7 and 8 are not observed with Holling's disc equation in this study or in the bifurcation diagram presented by Kuznetsov (2004). More complex dynamics than phase portraits 1 to 3 occur for roughly $\log _{10}\left(M_{\text {pred }} /\right.$ $\left.M_{\text {prey }}\right) \in[-0.5,0.5]$ and $1 / \omega>5$ with Ivlev's functional response. This range of body mass ratios corresponds to prey and predator species with similar body mass and metabolic rates, as we assume allometric scaling relationships. This range widens as prey carrying capacity increases.

In terms of structural sensitivity, there are two main differences between the bifurcation diagrams obtained for Holling's and Ivlev's functional response. The following results are only quantitatively modified by changes in the size of the parameter space investigated (Table 2). First, there are multiple attractors in $0.1 \%$ to $14.3 \%$ of the bifurcation diagram with Ivlev's functional response (see Section 3 in supplementary material for technical details). This proportion increases if higher $1 / \omega$ values (i.e. richer environment) are considered. Second, there is a stable limit cycle (phase portrait 2) with Holling's disc equation and a stable equilibrium (phase portrait 1) with Ivlev's functional response in $26.0-49.4 \%$ of the parameter space explored. This proportion decreases if higher $1 / \omega$ values are considered. The opposite situation between functional responses occurs only in a neglectible proportion of the parameter space here (near the Cusp and Bogdanov-Takens points). The order of magnitude of these two main results remains similar if any other parameter value is changed by $\pm 20 \%$ (see Table 3 ). Depending on the parameter, quantitative changes in our results can be of different amount. However, this impact of each parameter will not be discussed here as the analysis presented in Table 3 is exploratory.

\section{Generalized predator-prey model: beyond a specific formulation}

In the previous section, we have studied the effect of functional response formulation change on a predator-prey model. Now we use the generalized modelling approach (Gross and Feudel, 2006) to provide another way to interpret the stability of positive equilibria independently of a specific functional response formulation. This helps to understand differences between bifurcation diagrams obtained from different formulations. Model (7) also exhibits limit cycles. Like for equilibria, their stability can be studied through generalized modelling. However, the amount of technical work required (even for a predator-prey model without predator competition, Kuehn and Gross, 2011) is beyond the scope of this paper.

Table 2

Impact of the size of the parameter space explored on results of the bifurcation analysis.

\begin{tabular}{|c|c|c|c|c|}
\hline & \multirow[t]{2}{*}{ Reference value } & \multirow[t]{2}{*}{ Obtained range } & \multicolumn{2}{|c|}{ Sensitivity to explored parameter values } \\
\hline & & & $1 / \omega$ & $\log _{10}\left(M_{\text {pred }} / M_{\text {prey }}\right)$ \\
\hline Limit cycle vs. equilibrium & 0.359 & {$[0.260,0.494]$} & -0.0084 & NS \\
\hline Multiple attractors & 0.019 & {$[0.001,0.143]$} & 0.0055 & $-0.0024(p$-value $=0.07)$ \\
\hline
\end{tabular}

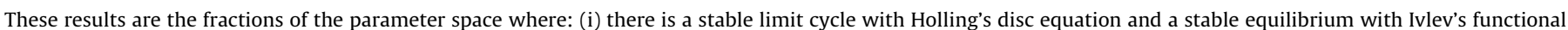

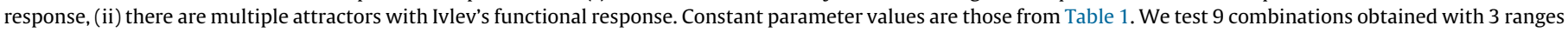

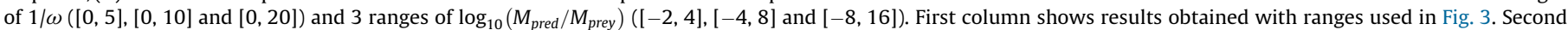

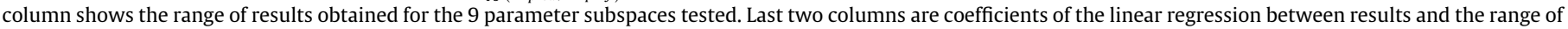
both parameters. NS means non-significant ( $p$-value $\gg 0.05)$. Coefficients for $1 / \omega$ are significant $(p$-value $<0.05)$. 
Table 3

Impact of other parameter values on results of the bifurcation analysis.

\begin{tabular}{|c|c|c|c|c|c|}
\hline \multirow[t]{2}{*}{ Biological meaning } & \multirow[t]{2}{*}{ Parameter } & \multicolumn{2}{|c|}{ Limit cycle vs. equilibrium } & \multicolumn{2}{|c|}{ Multiple attractors } \\
\hline & & $-20 \%$ & $+20 \%$ & $-20 \%$ & $+20 \%$ \\
\hline Mortality rate & $\alpha$ & $-0.7 \%$ & $-0.3 \%$ & $+50.3 \%$ & $+45.9 \%$ \\
\hline per-capita competition rate & $\beta$ & $-5.4 \%$ & $-4.0 \%$ & $+0.5 \%$ & $+49.9 \%$ \\
\hline Attack rate & $a^{H}$ & $-5.3 \%$ & $-2.7 \%$ & $+52.0 \%$ & $-18.9 \%$ \\
\hline Handling time & $h^{H}$ & $-1.0 \%$ & $0 \%$ & $+37.4 \%$ & $-46.3 \%$ \\
\hline Assimilation efficiency & $\lambda$ & $+3.5 \%$ & $-4.9 \%$ & $-77.3 \%$ & $+107.4 \%$ \\
\hline Resource biomass & $B_{\text {res }}$ & $0 \%$ & $0 \%$ & $+0.1 \%$ & $+0.1 \%$ \\
\hline
\end{tabular}

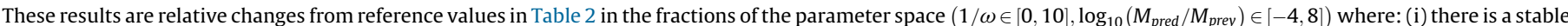

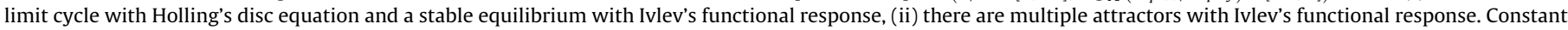

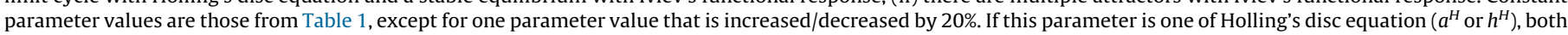

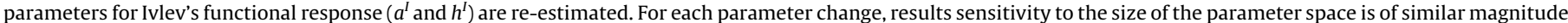
as in Table 2 .

We first consider the differential system (7) without specifying the functional response and the parameter values. We only claim that the functional response fulfills properties (2). So we consider in fact a family of models. Generalized modelling supposes that some models in this family have positive equilibria. The stability of a positive equilibrium is then studied using the Jacobian matrix:
Details on the derivation procedure to define generalized parameters are presented in Gross and Feudel (2006) and Yeakel et al. (2011). Equilibrium stability is studied as a function of the generalized parameter values without specific assumptions on the functions and original parameter (such as $a^{\phi}$ or $h^{\phi}$ ) values behind them.

$J=\left(\begin{array}{cc}\lambda q^{\phi}-B_{\text {pred }}^{*} \frac{M_{\text {pred }}^{-0.25}}{M_{\text {prey }}^{-0.25}} G_{-}^{\phi^{\prime}}\left(B_{\text {prey }}^{*}\right)-\left(\alpha+2 \omega \beta B_{\text {prey }}^{*}\right) & -G_{*}^{\phi *} \\ \lambda B_{\text {pred }}^{*} \frac{M_{\text {pred }}^{-0.25}}{M_{\text {prey }}^{-0.25}} G^{\phi^{\prime}}\left(B_{\text {prey }}^{*}\right) & \left(\lambda G_{*}^{\phi *}-\left(\alpha+2 \beta B_{\text {pred }}^{*}\right)\right) \frac{M_{\text {pred }}^{-0.25}}{M_{\text {prey }}^{-0.25}}\end{array}\right)$

evaluated at a positive equilibrium $\left(B_{\text {prey }}^{*}, B_{\text {pred }}^{*}\right)$, with $G^{\phi *}:=G^{\phi}\left(B_{\text {prey }}^{*}\right)$. The matrix is expressed using generalized parameters that describe the system close to this equilibrium (Table 4):

$J=\left(\begin{array}{cc}\tau_{\text {prey }}\left(1-\left(1-\delta_{\text {prey }}\right) \mu_{\text {prey }}-\delta_{\text {prey }} \gamma_{\text {pred }}\right) & -\tau_{\text {prey }} v_{\text {prey }, \text { pred }} \delta_{\text {prey }} \\ \tau_{\text {pred }} v_{\text {pred } \text { prey }} \gamma_{\text {pred }} & \tau_{\text {pred }}\left(1-\mu_{\text {pred }}\right)\end{array}\right)$.

\section{Table 4}

Formulation and ecological meaning of the generalized parameters used to build the generalized predator-prey model.

\begin{tabular}{|c|c|}
\hline Generalized parameter formulation & Ecological meaning \\
\hline Scale parameters & \\
\hline $\begin{aligned} \tau_{\text {prey }} & =\lambda q^{\phi}=G_{*}^{\phi *} \frac{B_{\text {pred }}^{*}}{B_{\text {prey }}^{*}} \frac{M_{\text {pred }}^{-0.25}}{M_{\text {prey }}^{-0.25}}+\left(\alpha+\omega \beta B_{\text {prey }}^{*}\right) \\
\tau_{\text {pred }} & =\lambda G^{\phi *} \frac{M_{\text {pred }}^{-0.25}}{M_{\text {prey }}^{-0.25}}=\left(\alpha+\beta B_{\text {pred }}^{*}\right) \frac{M_{\text {pred }}^{-0.25}}{M_{\text {prey }}^{-0.25}}\end{aligned}$ & $\begin{array}{l}\text { Time scales of species } \\
\text { dynamics }\end{array}$ \\
\hline$\delta_{\text {prey }}=\frac{1}{\tau_{\text {prey }}} G_{*}^{\phi *} \frac{B_{\text {pred }}^{*}}{B_{\text {prey }}^{*}} \frac{M_{\text {pred }}^{-0.25}}{M_{\text {prey }}^{-0.25}}$ & $\begin{array}{l}\text { Fraction of prey losses } \\
\text { due to predation }\end{array}$ \\
\hline $\begin{array}{l}1-\delta_{\text {prey }}=\frac{1}{\tau_{\text {prey }}}\left(\alpha+\omega \beta B_{\text {prey }}^{*}\right) \\
v_{\text {prey,pred }}=B_{\text {prey }}^{*} / B_{\text {pred }}^{*} \\
v_{\text {pred,prey }}^{*}=B_{\text {pred }}^{*} / B_{\text {prey }}^{*}\end{array}$ & $\begin{array}{l}\text { Fraction of prey losses } \\
\text { due to intrinsic dynamics } \\
\text { Biomass ratios between } \\
\text { species }\end{array}$ \\
\hline $\begin{array}{l}\text { Elasticities (also called exponent parameters) } \\
\mu_{\text {prey }}=1+\frac{\omega \beta B_{\text {prey }}^{*}}{\alpha+\omega \beta B_{\text {prey }}^{*}} \in[1,2] \\
\mu_{\text {pred }}=1+\frac{\beta B_{\text {pred }}^{*}}{\alpha+\beta B_{\text {pred }}^{*}} \in[1,2]\end{array}$ & $\begin{array}{l}\text { Non-linearity of species } \\
\text { intrinsic mortality }\end{array}$ \\
\hline $\begin{aligned} \gamma_{\text {pred }} & =g^{\phi^{\prime}}(1) \in[0,1] \text { with } b_{\text {prey }}=\frac{B_{\text {prey }}}{B_{\text {prey }}^{*}} \\
& \text { and } g^{\phi}\left(b_{\text {prey }}\right):=\frac{G^{\phi}\left(b_{\text {prey }} B_{\text {prey }}^{*}\right)}{G^{\phi}\left(B_{\text {prey }}^{*}\right)}\end{aligned}$ & $\begin{array}{l}\text { Slope of predator normalized } \\
\text { functional response }\end{array}$ \\
\hline
\end{tabular}

The scale parameters describe the time scale of species dynamics (for $\tau_{i}$ ) and the relative contribution of the different processes to this dynamics. Elasticities measure the non-linearity of processes.
To study equilibrium stability, let us recall the following statements (that hold under nondegeneracy conditions) from bifurcation theory (see for example Guckenheimer and Holmes, 1983; Perko, 1996; Kuznetsov, 2004). A saddle-node bifurcation occurs when there is a real zero eigenvalue. A common way to track this bifurcation is to solve $\Psi_{S N}=0$, with

$$
\begin{aligned}
\Psi_{S N}:=\operatorname{det}(J)= & \tau_{\text {prey }} \tau_{\text {pred }}\left[\left(1-\mu_{\text {pred }}\right)\left(1-\left(1-\delta_{\text {prey }}\right) \mu_{\text {prey }}\right)\right. \\
& \left.+\mu_{\text {pred }} \delta_{\text {prey }} \gamma_{\text {pred }}\right]
\end{aligned}
$$

vanishing only when at least one eigenvalue is equal to zero. A Hopf bifurcation occurs when there is a pair of (conjugate) pure imaginary eigenvalues. A common way to track this bifurcation in planar systems is to solve $\Psi_{H}=0$ with

$$
\Psi_{H}:=\operatorname{Tr}(J)=\tau_{\text {prey }}\left[1-\left(1-\delta_{\text {prey }}\right) \mu_{\text {prey }}-\delta_{\text {prey }} \gamma_{\text {pred }}+\frac{\tau_{\text {pred }}}{\tau_{\text {prey }}}\left(1-\mu_{\text {pred }}\right)\right] \text {. }
$$

Note that $\Psi_{H}$ vanishes at a Hopf bifurcation, but as well as if there are two real eigenvalues of opposite sign and same absolute magnitude. In this case the equilibrium is called a neutral saddle. The two situations can be discriminated by looking at the sign of $\Psi_{S N}$ which is positive at a Hopf bifurcation and negative at a neutral saddle. The limit case between these two situations corresponds to the intersection between a Hopf and a saddle-node bifurcation. It is a codimension-two Bogdanov-Takens bifurcation where two eigenvalues are equal to zero.

A five-dimensional bifurcation diagram is required to explore the whole parameter space. Fig. 4 displays three-dimensional diagrams that are sufficient to understand the role of all parameters, except for time scales $\tau_{\text {prey }}$ and $\tau_{\text {pred }}$. The ratio $\tau_{\text {pred }} /$ $\tau_{\text {prey }}$ has only a scaling impact proportional to $\mu_{\text {pred }}$ values on the Hopf bifurcation location, as it can be understood by looking at $\Psi_{H}$ equation (12). An equilibrium is stable when prey losses are dominated by density-dependent mortality (low $\delta_{\text {prey }}$ and high $\left.\mu_{\text {prey }}\right)$ and when prey density is sufficiently low to give a high slope 
(a)

non-linearity of predator mortality $\mu_{\text {pred }}=1$ (b)

non-linearity of predator mortality $\mu_{\text {pred }}=1.2$

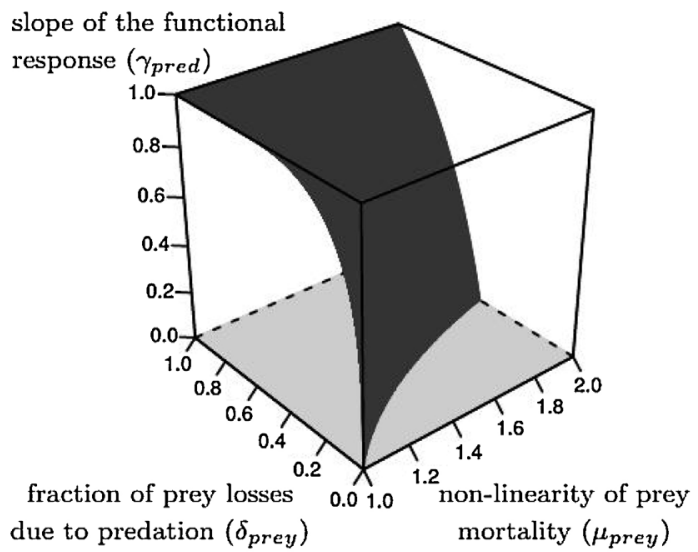

(c)

non-linearity of predator mortality $\mu_{p r e d}=1.4$

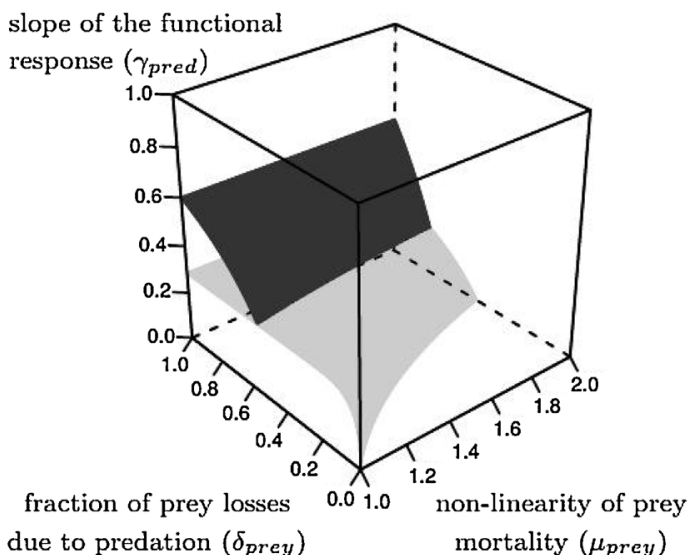

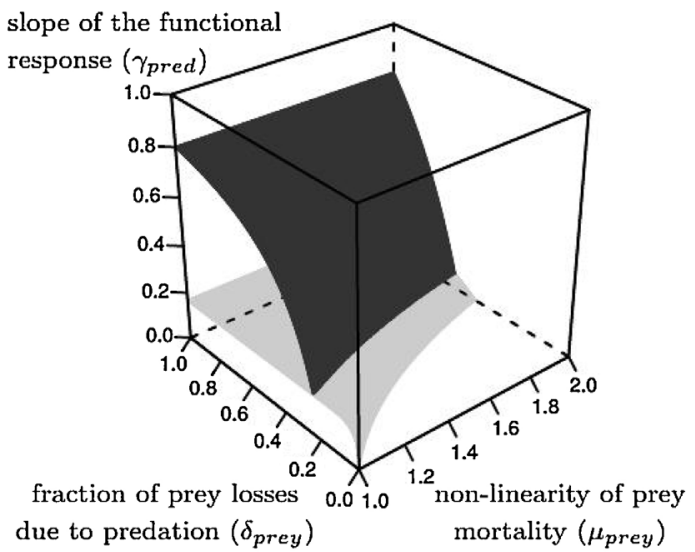

(d)

non-linearity of predator mortality $\mu_{\text {pred }}=1.6$

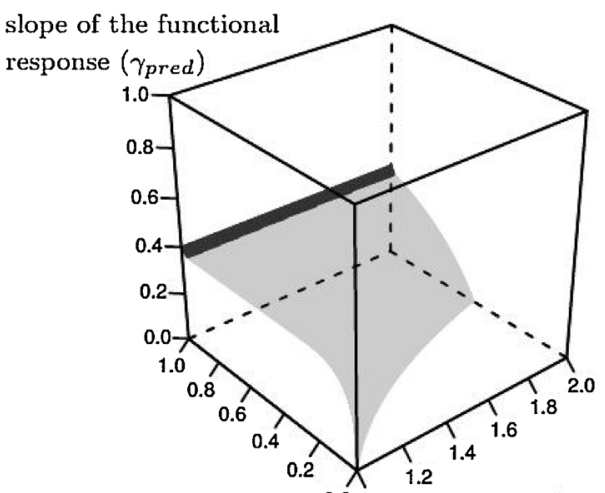

fraction of prey losses 0.01 .0 non-linearity of prey due to predation $\left(\delta_{\text {prey }}\right) \quad$ mortality $\left(\mu_{\text {prey }}\right)$

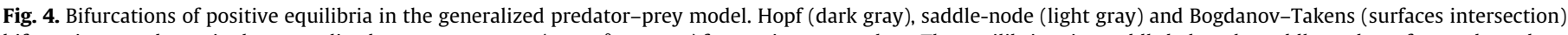

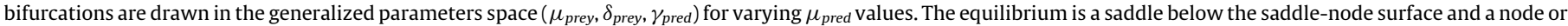

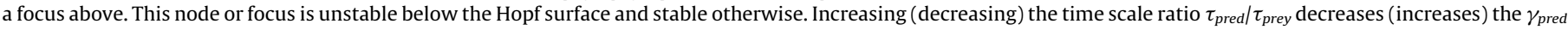

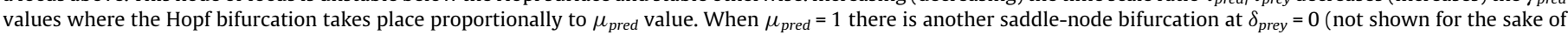

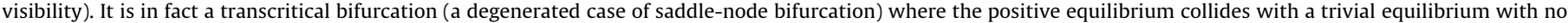
predator and disappears.

to the predator functional response (high $\gamma_{\text {pred }}$ ). For $\mu_{\text {pred }}=1$, an unstable equilibrium is always a node or a focus. As the densitydependence of predator mortality increases ( $\mu_{\text {pred }}$ increases), it can be a saddle for an increasing range of $\gamma_{\text {pred }}$ values. For sufficiently high $\mu_{\text {pred }}$, an equilibrium is either a stable node (or focus) or a saddle. These results hold for any positive equilibrium of any predator-prey model of the form (7).

Axes in Fig. 4 correspond to generalized parameters describing a positive equilibrium. Numerical values of the generalized parameters can be computed for all positive equilibria in the bifurcation diagrams of Fig. 3. By construction, the stability of these equilibria can be interpreted in terms of generalized parameters by mapping their numerical values in the bifurcation diagram of the generalized model (Fig. 4). So, when there are two or more positive equilibria in a specific model such as system (7), each equilibrium has a different generalized parameter set. For example, consider parameter values in Fig. 3 close to a saddle-node bifurcation, and where a node (or focus) and a saddle coexist. If generalized parameter values are computed and mapped in Fig. 4, the node is above the saddle-node bifurcation surface and the saddle is below. If a parameter of model (7) is moved towards the bifurcation in Fig. 3, generalized parameter values of both equilibria become closer. These values become equal to the saddle-node bifurcation value in Fig. 4 where equilibria collide and disappear, as the parameter of model (7) reaches the saddle-node bifurcation in Fig. 3.

Now we use generalized parameters to interpret the impact of the functional response formulation in Fig. 3. Equilibria are computed on a grid by steps of 0.02 for $\log _{10}\left(M_{\text {pred }} / M_{\text {prey }}\right)$ and 0.01 for $1 / \omega$. Consider values of these two parameters where there is a stable positive equilibrium (phase portrait 1) with Ivlev's functional response and a stable limit cycle (phase portrait 2) with Holling's disc equation. Generalized parameters are computed for the stable equilibrium with Ivlev's functional response and for the unstable focus surrounded by the limit cycle with Holling's disc equation. On average within the considered area, higher $\mu_{\text {prey, }}$ $\mu_{\text {pred }}$ and $\gamma_{\text {pred }}(\approx+0.1)$ as well as lower $\delta_{\text {prey }}(\approx-0.2)$ are obtained with Ivlev's functional response. The direction of these changes 


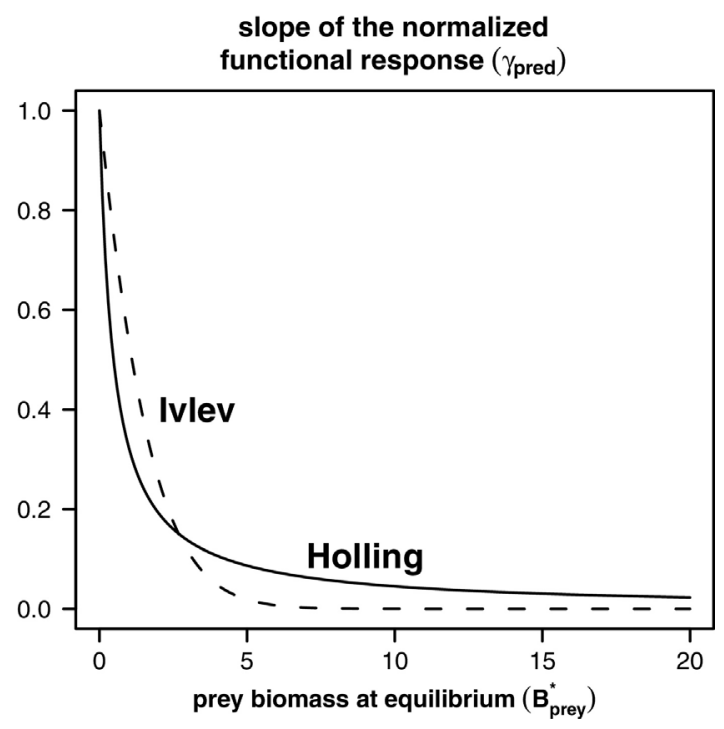

Fig. 5. Slope of the normalized functional responses $\left(\gamma_{\text {pred }}\right)$ used in the model: Holling's disc equation (solid) or Ivlev's functional response (dashed).

makes the equilibrium moving from the unstable part of the generalized parameter space (Fig. 4) with Holling's disc equation to the stable part with Ivlev's functional response by crossing the Hopf bifurcation. The increase of $\mu_{\text {prey }}$ and $\mu_{\text {pred }}$ can be explained by higher prey and predator biomasses at equilibrium $(\approx+0.1)$ with Ivlev's functional response, and thus a higher intra-specific competition. As a consequence, a lower fraction of prey losses are due to predation (lower $\delta_{\text {prey }}$ ). The predator has a stronger response (higher increase of the predation flux) to an increase in prey biomass, as the slope of the functional response near equilibrium is higher with Ivlev's functional response (higher $\left.\gamma_{\text {pred }}\right)$. Indeed, with this function, the functional response slope is higher within the range of prey biomass at the equilibrium $(\approx[0,2]$, Fig. 5).

\section{Discussion}

\subsection{Structural sensitivity and model predictions}

This paper shows that the number of attractors (or stable states) is modified if a slight change of functional response formulation occurs. Independently of the functional response formulation, the use of predator quadratic mortality leads to complex system dynamics. Indeed, seven of the twelve generic phase portraits of the predator-prey system display two or three attractors (Fig. 2). Multiple attractors are found in less than $0.001 \%$ of the parameter space studied with Holling's disc equation and in $1.9 \%$ with Ivlev's functional response (Fig. 3). Fussmann and Blasius (2005) briefly reported a similar situation for Rosenzweig \& MacArthur's model. This model has one attractor with Holling's and Ivlev's functional responses, but can have two attractors (an equilibrium and a limit cycle) when the functional response is a hyperbolic tangent function.

Changes in the number of attractors come in addition to changes in attractor type (equilibrium vs. limit cycle). These changes are partly due to differences in local slope of the functional response, as it has been previously reported (Oaten and Murdoch, 1975; Myerscough et al., 1996; Fussmann and Blasius, 2005; Cordoleani et al., 2011; Yeakel et al., 2011; Adamson and Morozov, 2012). We highlighted this effect by studying a generalized predator-prey model. A continuous change in model formulation makes the model exhibits a Hopf bifurcation which destabilizes the equilibrium and gives birth to a stable limit cycle. This bifurcation explains why in $35.9 \%$ of the parameter space explored, the (unique) attractor is a limit cycle with Holling's disc equation and an equilibrium with Ivlev's functional response (Fig. 3).

Both effects of functional response formulation (number and kind of attractor(s)) interact to create different system dynamics (Fig. 3). So depending on the formulation, the model can predict very different system responses to disturbances. Disturbances can affect either a parameter or a state variable (Beisner et al., 2003). Fig. 6 shows an example of model predictions with changing prey carrying capacity due to prey resource(s) and/or habitat alteration. Similarly, Fig. 7 shows another example with changes in prey biomass like a spike of harvesting or introduction of individuals. In both examples, Holling's disc equation predicts a higher system resilience. Indeed, Ivlev's functional response predicts that disturbance puts the system in an alternative stable state. Escaping this alternative state requires a larger modification than going backward (hysteresis phenomena). Such hysteresis phenomena are not predicted by Holling's disc equation because the model has only one attractor with this formulation. In these two examples, state variable and parameter alterations, the system is still deterministic. If a stochastic effect like individual variability was included, a perturbation driving one population close to zero (once or frequently) could lead to species extinction(s).

We studied structural sensitivity by considering two classical type-II functional responses: Holling (1959b, 1965) and Ivlev (1955). A mixed functional response $G^{x}:=x G^{H}+(1-x) G^{I}$ can be built to continuously switch between formulations (Cordoleani et al., 2011). Bifurcations that occur during this continuous switch (through the mixing parameter $x$ ) explain differences between bifurcation diagrams obtained for different formulations. Nevertheless, the bifurcation analysis of the predator-prey model becomes 3-dimensional with respect to parameters (Aldebert et al., in prep), which is beyond the scope of this paper.

In order to quantify structural sensitivity, Cordoleani et al. (2011) compared a distance between model formulations and a distance between model outputs. The strength of this approach is to quantify both quantitative and qualitative changes of the stable state. In the light of our findings, this approach can be extended to quantify different aspects of structural sensitivity, such as changes in the number of stable states and system resilience in situations with hysteresis phenomena (Aldebert et al., in prep).

\subsection{Combining generalized and specific models}

Using a generalized predator-prey model, we found that equilibria in the considered class of model are stabilized by (i) a high slope of the functional response, and (ii) losses mainly driven by density-dependent per-capita intrinsic mortality for both species. In the limit case where predator mortality is linear, these results are consistent with those obtained by Yeakel et al. (2011) in a similar model. As stated in the section on generalized modelling, up to two limit cycles can exist in Bazykin's model, which exhibits global bifurcations (limit point of cycles, homoclinic loops). However, the study of periodic orbits using generalized models is more difficult, even for predator-prey models (Kuehn and Gross, 2011).

We want to highlight the usefulness of studying both a generalized model and specific models belonging to the family represented by the generalized model. The generalized model provides local but generic results on equilibria stability. On the other hand, a specific model provides a specific but global understanding of system dynamics, including periodic orbits and the co-existence of multiple attractors. The generalized model helps to understand the specific model. We used this framework to understand the effects of changing the functional response 


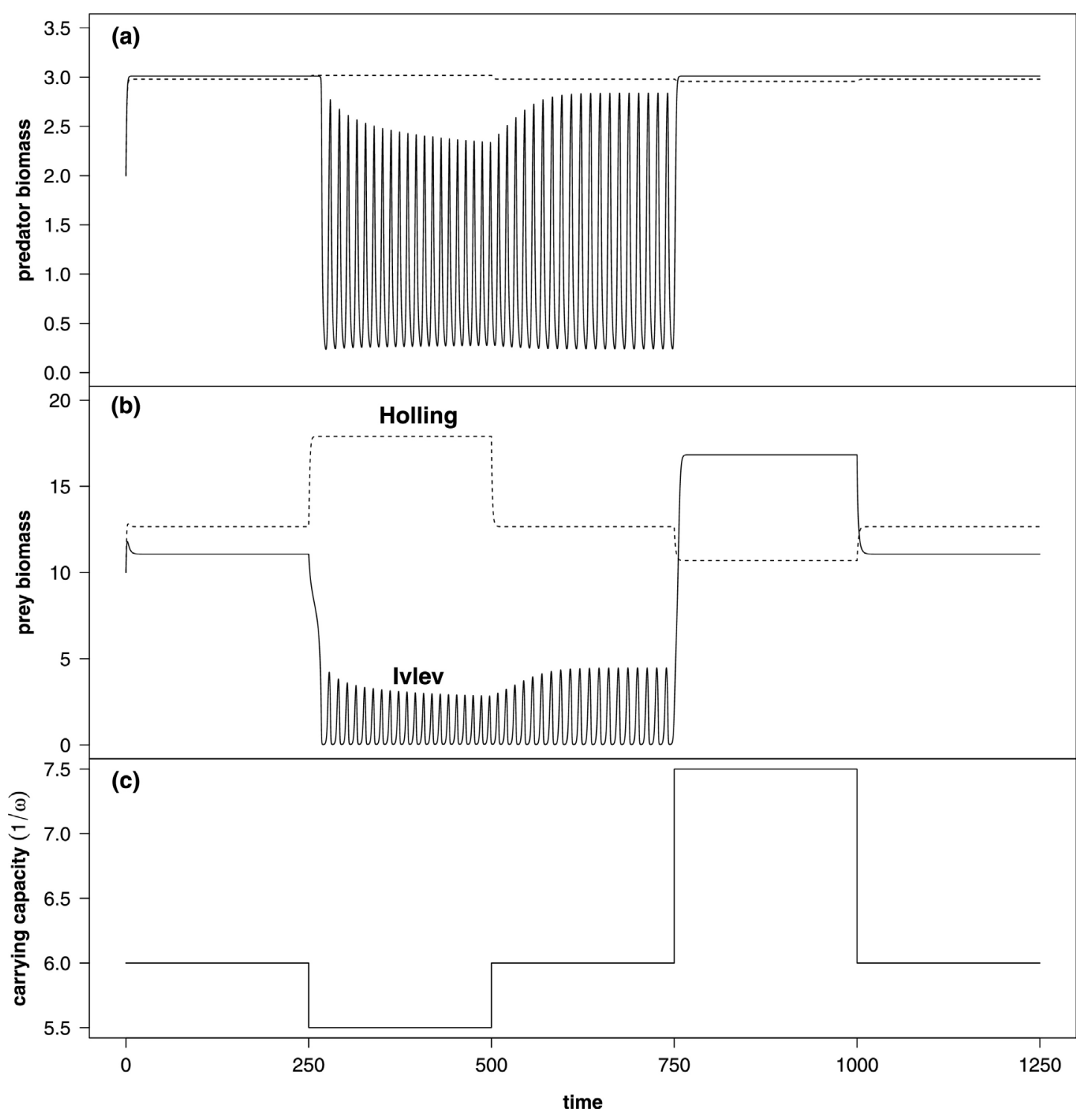

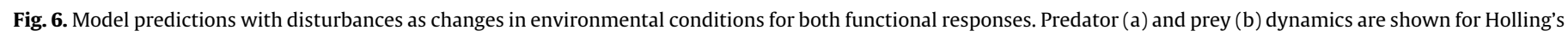

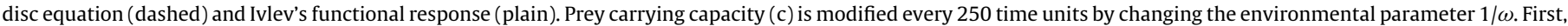

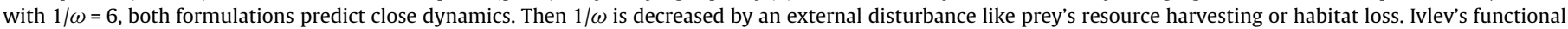

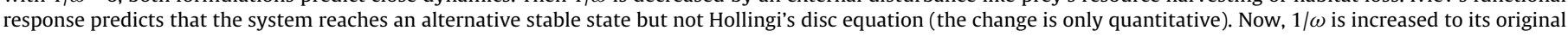

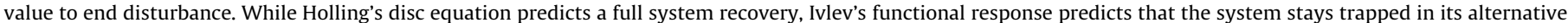

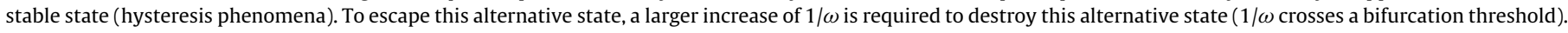

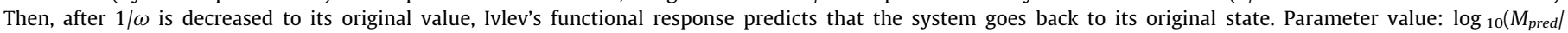
$\left.M_{\text {prey }}\right)=0.45$. Initial conditions: $B_{\text {prey }}(0)=10, B_{\text {pred }}(0)=2$.

formulation on equilibria stability. At the opposite, the specific model also helps the interpretation of the generalized model (as also argued by Yeakel et al., 2011). For example, a saddle-node bifurcation in the generalized model is in fact a transcritical bifurcation (a degenerated case of saddle-node bifurcation) after which the positive equilibrium disappears (see Fig. 4). Indeed, with the generalized model we supposed that a positive equilibrium exists and thus cannot focus on its existence conditions. For example, a high slope of the functional response means a higher stability of positive equilibria, but also low prey biomass and potentially predator extinction. This last point cannot be captured by generalized modelling. Positive equilibria with such low prey biomass can exist for some specific models represented by the generalized model. Nevertheless, the distribution of generalized parameter values among all specific models may not be uniform.

From a biological point of view, a specific model allows more insights as processes are explicitly modelled, but a generalized model requires less knowledge of the system to model. Indeed, generalized modelling describes processes near equilibrium, without specific assumptions on these processes far from this focal equilibrium. In the example of functional response, the generalized model needs only a knowledge of its slope near equilibrium, which can be estimated from empirical data. However, this knowledge allows to predict only system dynamics near equilibrium. Knowing system dynamics far from this equilibrium can be of interest to predict system response to external disturbances (like in Figs. 6 and 7). Such dynamics can be figured out under the assumption that they might be quite simple, for example that there is only one equilibrium. However, more complex dynamics cannot be figured out from the local knowledge of the system near equilibrium. A deeper knowledge of the system is required, like an accurate functional response formulation. In this situation, we leave the generic approach of generalized modelling to study a specific system based on more restrictive 


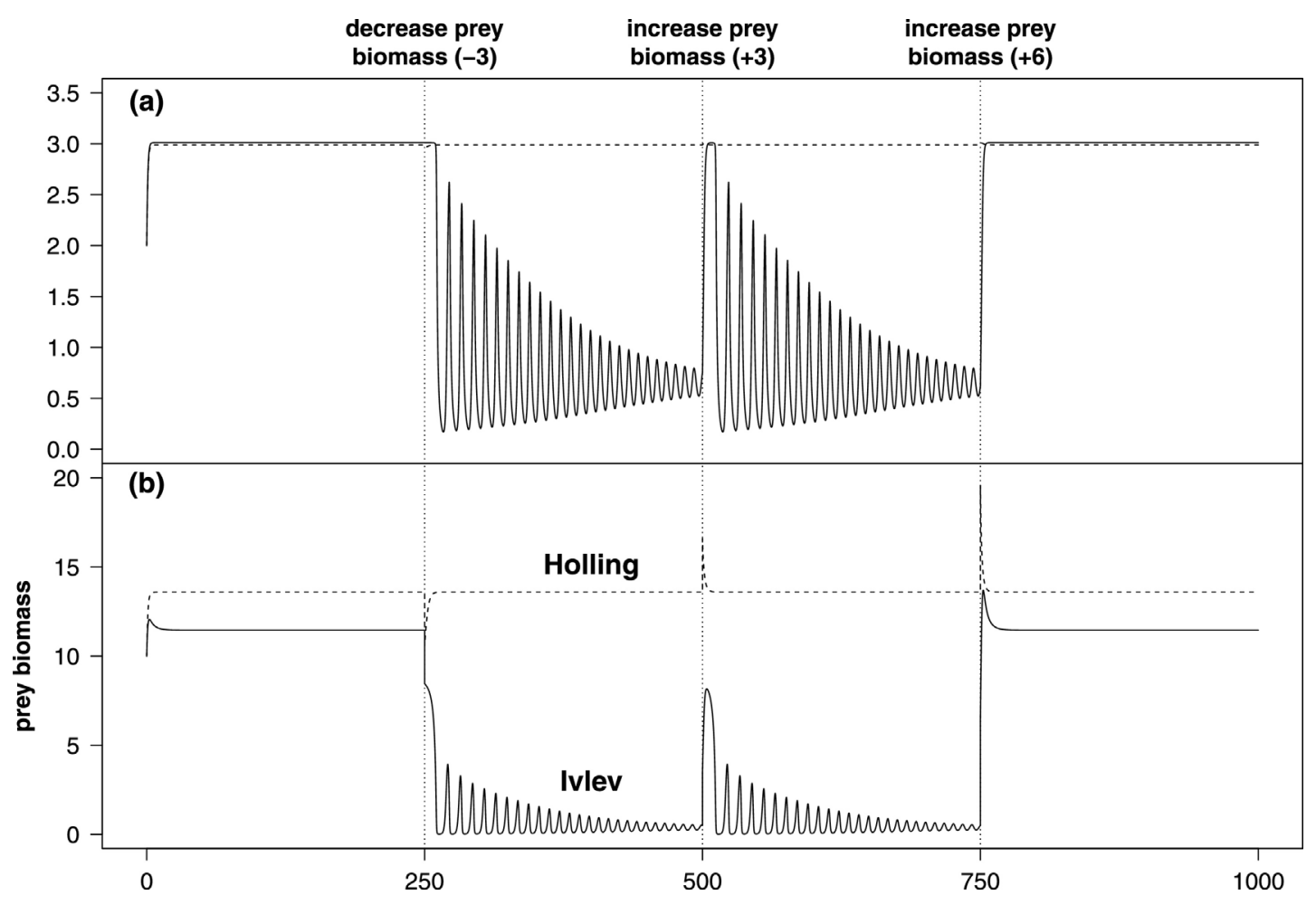

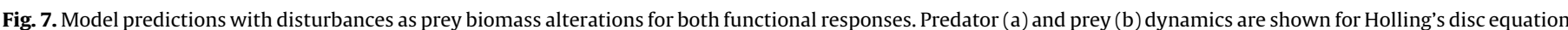

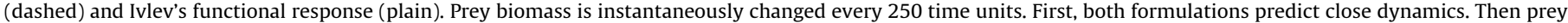

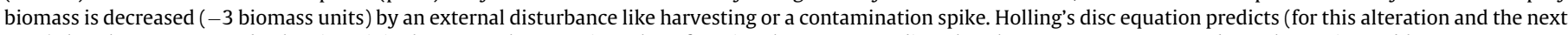

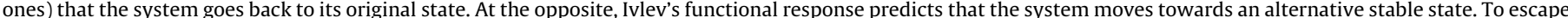

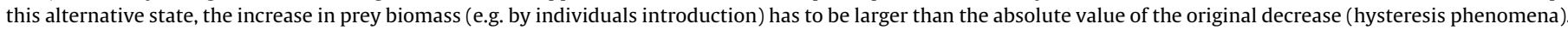
Parameter values: $1 / \omega=6.7, \log _{10}\left(M_{\text {pred }} / M_{\text {prey }}\right)=0.2$. Initial conditions: $B_{\text {prey }}(0)=10, B_{\text {pred }}(0)=2$.

assumptions. How far these restrictive assumptions are accurate in the context of the system to model, and how uncertainties in these assumptions influence model predictions, are questions that lead to the study of structural sensitivity.

\section{Conclusion}

We investigated structural sensitivity in a predator-prey model with density-dependent mortality using a bifurcation analysis. The bifurcation diagram, drawn with respect to prey carrying capacity and species body mass ratio, is modified by the choice functional response formulation between close ones in two ways. First, there is a unique stable limit cycle in a smaller part of the parameter space with Ivlev's formulation than with Hollin's disc equation. Using generalized modelling, we highlighted the importance of the slope of the functional response in this difference, as it has been previously reported for simpler predator-prey and food chain models. Secondly, with Ivlev's functional response, dynamics with multiple attractors occur in a significant part of the parameter space where only one attractor exists with Holling's disc equation. With Ivlev's functional response there can be multiple stable states and hysteresis when disturbance appears, whereas there is no such situation with Holling's disc equation.

Because of intrinsic data variability and because model formulation is always a simplified representation of complex biological processes, the choice of the functional response remains uncertain. Here we showed that this uncertainty in the formulation can lead to uncertainties in model predictions through (i) the kind of stable state (equilibrium vs. fluctuations) the system will reach, (ii) the number of alternative stable states, and as a consequence, (iii) the system resilience in case of external disturbances. This work rises questions about the choice of functional response formulation in a given situation. For example, how to select a function with enough mechanistic basis to be more relevant than other ones, while being sufficiently simple and generic? Other questions are: (i) how much complex ecological models can be affected by this sensitivity to model formulation? and (ii) how to deal with these uncertainties in model predictions? This last point can be a challenging way of research for a better assessment of model uncertainties and thus more accurate predictions.

\section{Acknowledgements}

The authors acknowledge four anonymous reviewers for their very constructive comments on earlier versions of the manuscript. We thank Bob Kooi for fruitful discussions on bifurcation theory. We acknowledge Natascha Schmidt and Bastien Bellemin-Noel for language proof-reading. The PhD scholarship of C.A was funded by the French Ministry for Education and Research.

\section{Appendix A. Supplementary Data}

Supplementary data associated with this article can be found, in the online version, at http://dx.doi.org/10.1016/j.ecocom.2016.05. 004.

\section{References}

Adamson, M.W., Morozov, A.Y., 2012. When can we trust our model predictions? Unearthing structural sensitivity in biological systems.. Proc. R. Soc. Lond. A 469 (2149) .

Adamson, M.W., Morozov, A.Y., 2014. Defining and detecting structural sensitivity in biological models: developing a new framework. J. Math. Biol. 69, 18151848. 
Aldebert, C, Nerini, D, Gauduchon, M, Poggiale, JC. Does structural sensitivity alter complexity-stability relationships? Ecol. Complex., in preparation.

Anderson, T.R., Gentleman, W.C., Sinha, B., 2010. Influence of grazing formulations on the emergent properties of a complex ecosystem model in a global ocean general circulation model. Progress Oceanogr. 87, 201-213.

Arditi, R., Ginzburg, L.R., 1989. Coupling in predator-prey dynamics: ratio-dependence. J. Theor. Biol. 139, 311-326.

Bazykin, A.D., Kuznetsov, Yu A., Khibnik, A.I., 1985. Bifurcation diagrams of planar dynamical systems. Research Computing Center, USSR Academy of Sciences, Pushchino, Moscow Region (in Russian).

Beddington, J.R., 1975. Mutual interference between parasites or predators and its effect on searching efficiency. J. Anim. Ecol. 44 (1), 331-340.

Beisner, B.E., Haydon, D.T., Cuddington, K., 2003. Alternative stable states in ecology. Front. Ecol. 1 (7), 376-382.

Brose, U., Williams, R.J., Martinez, N.D., 2006. Allometric scaling enhances stability in complex food webs. Ecol. Lett. 9, 1228-1236.

Brown, J.H., Gillooly, J.F., Allen, A.P., Savage, V.M., West, G.B., 2004. Toward a metabolic theory of ecology. Ecology 85, 1771-1789.

Cantrell, R.S., Cosner, C., 2001. On the dynamics of predator-prey models with the Beddington-DeAngelis functional response. J. Math. Anal. Appl. 257, 206-222.

Cordoleani, F., Nerini, D., Gauduchon, M., Morozov, A., Poggiale, J.C., 2011. Structural sensitivity of biological models revisited. J. Theor. Biol. 283, 82-91.

DeAngelis, D.L., 2013. The case for ratio-dependence in trophic ecology. Trends Ecol. Evol. 28, 259-260.

DeAngelis, D.L., Goldstein, R.A., O'Neill, R.V., 1975. A model for trophic interaction. Ecology 56, 881-892.

Dhooge, A., Govaerts, W., Kuznetsov, Yu A., Mestrom, W., Riet, A.M., Sautois, B. 2006. MATCONT and CL MATCONT: Continuation toolboxes in matlab, http:// matcont.ugent.be/.

Fulton, E.A., Smith, A.D.M., Johnson, C.R., 2003. Effect of complexity on marine ecosystem models. Mar. Ecol. Progress Ser. 253, 1-16.

Fulton, E.A., Smith, A.D.M., Johnson, C.R., 2003. Mortality and predation in ecosystem models: is it important how these are expressed? Ecol. Modell. 169, 157-178.

Fussmann, G.F., Blasius, B., 2005. Community response to enrichment is highly sensitive to model structure. Biol. Lett. 1 (1), 9-12.

Gentleman, W., Leising, A., Frost, B., Strom, S., Murray, J., 2003. Functional responses for zooplancton feeding on multiple resources: a review of assumptions and biological dynamics. Deep-Sea Res. II 50, 2847-2875.

Gross, T., Feudel, U., 2006. Generalized models as a universal approach to the analysis of nonlinear dynamical systems. Phys. Rev. E 73 (1), 016205.

Gross, T., Ebenhöh, W., Feudel, U., 2004. Enrichment and foodchain stability: the impact of different forms of predator-prey interaction. J. Theor. Biol. 227, 349-358.

Guckenheimer, J., Holmes, P., 1983. Nonlinear Oscillations, Dynamical Systems, and Bifurcations of Vector Fields. Springer, , pp. 459.

Heckmann, L., Drossel, B., Brose, U., Guill, C., 2012. Interactive effects of body-size structure and adaptative foraging on food-web stability. Ecol. Lett. 15, 243-250.

Holling, C.S., 1959a. The components of predation as revealed by a study of smallmammal predation of the European pine sawfly. Can. Entomol. 91 (7), 293-320.

Holling, C.S., 1959b. Some characteristics of simple types of predation and parasitism. Can. Entomol. 91 (7), 385-398.

Holling, C.S., 1965. The functional response of predators to prey density and its role in mimicry and population regulation. Memoirs Entomol. Soc. Can. 45, 3-60.
Ivlev, V.S, 1955. Experimental Ecology of the Feeding of Fishes. Pischepromizdat, Moscow 302 pp. (translated from Russian by D. Scott, Yale University Press, New Haven, 1961).

Jeschke, J.M., Kopp, M., Tollrian, R., 2002. Predator functional response: discriminating between handling and digesting prey. Ecol. Monogr. 72 (1), 95-112.

Kartascheff, B., Guill, C., Drossel, B., 2009. Positive complexity-stability relations in food web models without foraging adaptation. J. Theor. Biol. 259, 12-23.

Kartascheff, B., Heckmann, L., Drossel, B., Guill, C., 2010. Why allometric scaling enhances stability in food web models. Theor. Ecol. 3, 195-208.

Knowlton, N., 2004. Multiple "stable" states and the conservation of marine ecosystems. Progress Oceanogr. 60, 387-396.

Kooijman, S.A.L.M., 2010. Dynamic Energy Budget Theory for Metabolic Organisation, 3rd ed. Cambridge University Press, Cambridge, pp. 514.

Kuehn, C, Gross, T. 2011. Nonlocal generalized models of predator-prey systems. arXiv preprint, 1105.3662.

Kuznetsov, Yu A., 2004. Elements of Applied Bifurcation Theory, 2nd ed. Springer, New York, pp. 361.

Loreau, M., 2010. From Populations to Ecosystems: Theoretical Foundations for A New Ecological Synthesis. Princeton University Press, Princeton, pp. 297.

Metzler, W., Wischniewsky, W., 1985. Bifurcations of equilibria in Bazykin's predator-prey model. Math. Modell. 6, 111-123.

Mullin, M.M., Stewart, E.F., Fuglister, F.J., 1975. Ingestion by planktonic grazers as a function of food concentration. Limnol. Oceanogr. 20 (2), 259-262.

Myerscough, M.R., Darwen, M.J., Hogarth, W.L., 1996. Stability, persistence and structural stability in a classical predator-prey model. Ecological Modelling 89, $31-42$.

Oaten, O.A., Murdoch, W.W., 1975. Functional response and stability in predatorprey systems. Am. Natural. 109, 289-298.

Perko, L., 1996. Differential Equations and Dynamical Systems, 2nd ed. Springer, New York, pp. 525.

Plitzko, S.J., Drossel, B., Guill, C., 2012. Complexity-stability relations in generalized food-web models with realistic parameters. J. Theor. Biol. 306, 7-14.

Poggiale, J.C., Baklouti, M., Queguiner, B., Kooijman, SA.L.M., 2010. How far details are important in ecosystem modelling: the case of multi-limiting nutrients in phytoplankton-zooplankton interactions. Philos. Trans. R. Soc. London B 365, 3495-3507.

Rosenzweig, M.L., MacArthur, R.H., 1963. Graphical representation and stability conditions of predator-prey interaction. Am. Natural. 97 (895), 209-223.

Scheffer, M., de Boer, R.J., 1995. Implications of spatial heterogeneity for the paradox of enrichment. Ecology 76 (7), 2270-2277.

Scheffer, M., Bascompte, J., Brock, W.A., Brokvin, V., Carpenter, S.R., Dakos, V., Held, H., van Nes, E.H., Rietkerk, M., Sugihara, G., 2009. Early-warning signals for critical transitions. Nature 461, 53-59.

Scheffer, M., Carpenter, S.R., Lenton, T.M., Bascompte, J., Brock, W.A., Dakos, V., van de Koppel, J., van de Leemput, I.A., Levin, S.A., van Nes, E.H., Pascual, M., Vandermeer, J., 2012. Anticipating critical transitions. Science 338, 344-348.

Williams, R.J., Martinez, N.D., 2000. Simple rules yield complex food webs. Nature 404, 180-183.

Wood, S.N., Thomas, M.B., 1999. Super-sensitivity to structure in biological models. Proc. R. Soc. London B 266, 565-570.

Yeakel, J.D., Stiefs, D., Novak, M., Gross, T., 2011. Generalized modeling of ecological population dynamics. Theor. Ecol. 4, 179-194. 